\title{
La inteligencia emocional, social y el conocimiento tácito. Su valor en la vida cotidiana
}

Norma Contini ${ }^{1}$

\section{Resumen}

A partir del interrogante acerca de qué distingue a los sujetos exitosos de los menos exitosos en la vida diaria se definen diversos tipos de inteligencia no académica.

Como las pruebas de CI no han logrado dar plena respuesta a esta pregunta, en las últimas décadas se ha configurado un campo de investigación en torno a otras formas de inteligencia, no académicas. Se hace referencia a las inteligencias social, práctica, emocional y al conocimiento tácito, con el propósito de deslindar su significado y analizar sus puntos de solapamiento. Se destaca la vinculación de aquellas con la resolución de problemas de la vida cotidiana. Se profundiza en el concepto de inteligencia emocional y se analiza el estado actual de las investigaciones, poniendo énfasis en si son habilidades mentales o si están implicadas variables de personalidad.

Se concluye destacando que, a pesar de las limitaciones teóricas y metodológicas actuales, los estudios sobre el tema han tenido el mérito de ampliar la concepción acerca de las habilidades cognitivas y advertir que es imprescindible integrar las emociones y los procesos de razonamiento si se quiere conocer más cabalmente el comportamiento humano.

Palabras clave: inteligencia social - inteligencia práctica - inteligencia emocional conocimiento tácito.

\section{Abstract}

Several types of non academic intelligence are defined considering the question about what distinguishes the successfull persons from unsuccessfull persons in everyday life. In the recent decades has rised a wide field of research about others kinds of intelligence in attempt tha IQ test had no offered a good explanation for the mentioned question. Tacit Knowledge, Social Intelligence, Practical Intelligence and Emotional Intelligence are defined and the overlaped points of those definitions are analized. It is considered the relation between those kind of intelligence and the everyday life problem solving. The concept of emotional intelligence is analized considering: mental abilities and personalities traits models. The studies about non academic forms of intelligence

1. Dra. en Psicología. Especialista en Psicología Clínica (Sección Psicodiagnóstico). Universidad de Barcelona. Universidad Nacional de Tucumán. Argentina. Investigadora CIUNT. E-mail: nocon@unt.edu.ar 
Psicodebate 5. Psicología, Cultura y Sociedad

had enlarged the cognitive abilities conception. Those studies had pointed out the importance of linking reasoning procceses and the emotions in order to know better human behavior.

Key words: social intelligence - practical intelligence -emotional intelligence- tacit knowledge. 
La inteligencia emocional, social y el conocimiento tácito. Su valor en la vida cotidiana

\section{Introducción}

Los estudios sobre la inteligencia académica tienen una vasta trayectoria desde que Binet a comienzos del siglo XX diseñara su célebre prueba. Se ha podido demostrar que la inteligencia académica tiene elevadas correlaciones con el rendimiento escolar. Las últimas décadas de aquel siglo han sido testigos de sólidas investigaciones que refinaron métodos de evaluación de este tipo de inteligencia. La práctica clínica y psicopedagógica fue mostrando la complejidad del funcionamiento cognitivo de un sujeto y las dificultades para establecer pronósticos certeros sobre el éxito en la vida cotidiana a partir de las pruebas de CI. En tal sentido es frecuente observar sujetos con elevado CI que tienen dificultades en su desempeño en la vida diaria, mientras que otros, de CI modesto lo hacen sorprendentemente bien.

Estas evidencias clínicas nos inducen a plantear, siguiendo a Goleman $(2000,1995)$, acerca de cuáles factores entran en juego para producir un resultado u otro - éxito o fracaso en la vida cotidiana.

$\mathrm{Al}$ respecto, en las últimas dos décadas ha habido un creciente interés por las formas no académicas de la inteligencia. Así, se han utilizado distintos constructos para poder definirlas; la dificultad reside en que tales constructos se solapan. Uno es el de inteligencia práctica (Sternberg 1985, 1997). Otro concepto alternativo es el de inteligencia social (Cantor y Kihlstrom, 1987; Ford y Maher, 1998) o el de las inteligencias intrapersonal e interpersonal (Gardner, 1994, 1983, 1993), el de inteligencia emocional (IE) (Goleman, 2000, 1995; Salovey y Mayer, 1990) y el de Conocimiento Tácito (Polanyi, 1966; Neisser, 1979; Schön, 1983, Sternberg, 1985, 1997)

Jones y Day (1997) aseveran que existen semejanzas entre estos conceptos, puesto que la inteligencia práctica, la social y emocional tienen en común el hecho de focalizan en un conocimiento de tipo declarativo y procesal. Igualmente afirman que es flexible y que se centra en la solución de problemas que admiten más de una interpretación.

Se hará referencia a la IE, a las diferencias de ésta con la inteligencia académica y a sus puntos de solapamiento con la inteligencia práctica, la inteligencia social y el conocimiento tácito, tanto como a la importancia que han cobrado los estudios sobre estos tipos de habilidades. Tal relevancia aparece vinculada al impacto que parecen tener en la organización psicológica del sujeto, en la calidad de vida y en las posibilidades de ser más o menos exitoso en la vida cotidiana. Por este motivo, los estudios sobre habilidades prácticas se están convirtiendo en la corriente principal de las investigaciones en cognición.

\section{Inteligencia Social}

Thorndike (1929) definió a la inteligencia social como la habilidad para comprender a otros y para comportarse con sensatez con relación a aquellos.

Con posterioridad se ensayaron diversas definiciones, tales como la que señala que se trata de la habilidad para tener éxito con los demás (Moss y Hunt, 1927), o para tratar 
Psicodebate 5. Psicología, Cultura y Sociedad

con la gente (Hunt, 1928); serenidad en el trato con otros, según los estados y características de aquellos (Vernon,1933). Wedeck (1947) agrega que es la habilidad para juzgar correctamente los sentimientos, los estados de ánimo y las motivaciones de los demás.

Kosmitzki y John (1993) trataron de identificar los componentes que parecen centrales en las teorías implícitas de la inteligencia social. Así, incluyen variables cognitivas y conductuales. Entre las cognitivas señalan la posibilidad de tomar perspectiva de los hechos, comprender a la gente, conocer reglas sociales y apertura hacia los demás. Las variables conductuales incluyen la habilidad para tratar con otros, adaptabilidad social y calidez interpersonal.

El interés por estudiar los diversos componentes de la inteligencia social se ha visto en gran parte frustrado por las dificultades que tuvieron los investigadores en diseñar instrumentos que evaluaran de un modo diferenciado las habilidades sociales de la abstracta o académica. Esta dificultad está en directa relación con el solapamiento existente entre ambos tipos de inteligencia. En el fondo, la complejidad del tema reside en que se trata de un constructo multidimensional. Complementariamente, los obstáculos para hacer esta distinción obedecen a que se puso mucho énfasis en los aspectos cognitivos de la inteligencia social y en que los métodos de evaluación son predominantemente verbales.

A su vez, utilizando medidas no verbales de evaluación se pudo concluir (Barnes y Sternberg, 1989) que la habilidad para decodificar cuidadosamente la comunicación no verbal de un interlocutor, es un indicador relevante de inteligencia social. Wong, Day, Maxwell y Meara (1995) identificaron 3 aspectos cognitivos de la misma: a) conocimiento social (conocimiento de las reglas de cortesía); b) percepción social (habilidad para comprender el estado emocional de los demás); c) insight social (habilidad para comprender las conductas observadas en un contexto social)

Por otra parte, Cantor y Kihlstrom (1987) y Cantor y Harlow (1994) analizaron la personalidad desde la perspectiva de la inteligencia social. Señalan, desde esta óptica, que el comportamiento social es inteligente porque esta mediatizado por procesos cognitivos tales como la percepción, memoria, razonamiento y resolución de problemas.

Siguiendo esta línea de análisis, Cantor y Harlow han estudiado los procesos cognitivos que subyacen a la inteligencia social. De ese modo focalizan en tareas vitales, como hacer amigos, encontrar un cónyuge, definir una carrera u obtener buenas posiciones sociales. Para ello escogieron períodos de transición entre una etapa y otra del ciclo vital, como el paso de la escuela secundaria a la universidad. Encontraron que los sujetos formulan planes de acción y monitorean sus progresos y los resultados de aquellos. Agregan que, si los planes propuestos se ven obstaculizados, los sujetos son capaces de cambiarlos. Estos procesos identificados por Cantor y Harlow son coincidentes con los que Sternberg (1985) señala como subyacentes a la inteligencia exitosa.

Sternberg agrega que lamentablemente los esfuerzos en definir y evaluar la inteligencia social no han conducido a una mejor comprensión del constructo y que hay tantas definiciones como investigadores. A modo de síntesis, se puede decir que las definiciones hacen referencia a: 
La inteligencia emocional, social y el conocimiento tácito. Su valor en la vida cotidiana

- percepción social

- conocimiento social

- insight social

- empatía

- memoria social

- adaptación social.

\section{Inteligencia Práctica}

En general, los problemas han sido clasificados en académicos y prácticos (Neisser, 1979; Sternberg, 1985, 1997; Wagner y Sternberg,1985), pero no hay consenso acerca de cómo definir los problemas prácticos que se encuentran en la vida diaria.

Los sujetos afrontan habitualmente problemas cuya solución no se encuentra disponible a partir del conocimiento adquirido en el contexto escolar o académico. Estos tipos de problemas se experiencian en la vida diaria. La inteligencia práctica o de la vida cotidiana se presenta así, diferente de la inteligencia académica.

La inteligencia práctica se relaciona con la viveza en la calle o con el sentido común, mientras que la inteligencia académica supone operaciones formales del pensamiento sobre temas generalmente ajenos a la experiencia cotidiana del sujeto.

Personas exitosas en la escuela presentan déficit en sus relaciones sociales, o a la inversa, hay quienes fracasan en la escuela, pero tienen éxito en el trabajo o en los vínculos sociales.

Pareciera que la mayor fuente de diferencias está dada por la disparidad de problemas que consideran un tipo de inteligencia y otro. Así, las cuestiones con las que el sujeto se enfrenta en la vida diaria poco tienen que ver con las habilidades adquiridas en la escuela, por ejemplo la capacidad de un niño para realizar operaciones de potenciación o raíz cuadrada no se relacionan en absoluto con las habilidades que debe desplegar si su hermanito menor llora desconsoladamente de hambre, la madre está fuera de la casa y él debe proporcionarle alimento.

Los problemas académicos son aquellos que tienden a ser formulados por otros, no por el propio sujeto. Están bien definidos, proveen datos completos para su análisis y admiten una sola respuesta. Otra característica es que están fuera de la experiencia cotidiana.

Los problemas prácticos -en contraste con los académicos- tienden a estar pobremente formulados, son de interés personal y no siempre contienen todos los datos para su solución. Están directamente relacionados con la experiencia de la vida diaria y admiten múltiples soluciones.

Las habilidades que un sujeto pone de manifiesto para resolver problemas prácticos, al combinarse, dan como resultado lo que se conoce como Inteligencia Práctica. Es definida como aquella que facilita un mejor ajuste del sujeto al entorno, ya sea que éste se adapte al medio ambiente, o que lo modifique.

Sternberg señala, con mucho acierto, que las habilidades en las que enfatiza la escolarización formal tienen un valor muy limitado si no pueden ser empleadas en la 
Psicodebate 5. Psicología, Cultura y Sociedad

resolución de problemas de la vida diaria. En tal sentido las críticas a la escuela han sido demoledoras, puesto que parece no poder resolver la disociación entre el saber académico del aula, y el de la vida cotidiana.

No obstante reconocer las dificultades para evaluar la inteligencia social, se puede vislumbrar que el denominado Conocimiento Tácito representa un intento de evaluar las habilidades que se ponen en juego en contextos específicos, lo que puede ser considerado un aspecto de la inteligencia práctica (Cantor y Harlow, 1994).

\section{Conocimiento Tácito e Inteligencia Práctica}

Sternberg, Forsythe, Hedlund, Jorvath, Wagner, Williams, Snook, y Grigorenko (2000) se plantearon como pregunta de investigación qué es lo que distingue a los sujetos más exitosos de los menos exitosos en la vida diaria. Descubrieron que gran parte del conocimiento necesario para el éxito en actividades de la vida cotidiana era tácito. Ello quiere decir que se adquiere durante la ejecución de las tareas diarias pero sin que el sujeto tenga consciencia de lo que está aprendiendo.

Es Polanyi (1966) quien introduce el término y puede definirse como aquel que caracteriza al conocimiento obtenido de la experiencia diaria y que tiene una cualidad implícita y no articulada (Neisser, 1979, Sternberg, 1985, 1988, 1997).

Sternberg et al. (2000) consideran al conocimiento tácito como un aspecto de la inteligencia práctica. Refleja la habilidad para aplicar el conocimiento adquirido en la vida diaria al logro de objetivos o metas valoradas por el sujeto. Se considera que el conocimiento tácito es necesario para un ajuste exitoso al medio.

Una de las características salientes de este tipo de conocimiento es que se adquiere por cuenta propia, por la experiencia individual más que por la instrucción. Por lo tanto, tiende a no ser verbalizado, a no ser enfatizado y está explícita y pobremente relacionado con el éxito en la práctica.

Otra característica es su asociación con la acción, vale decir, es procesal. Toma la forma de saber cómo más que de saber qué. Es un conocimiento orientado hacia un uso en particular, en un contexto específico, tal como la escuela, el trabajo o la vida en el hogar, y en una situación dada, a través de la relación con un mentor o bien del propio sujeto como autodidacta. Sternberg agrega que cuando se interroga a los sujetos acerca de cómo adquirieron el conocimiento suelen hacer toscas declaraciones verbales sobre reglas generales, por ejemplo, si consultamos a un ama de casa cómo aprendió a hacer tan excelente tarta, dirá "estando en la cocina". Es como si el sujeto no fuera plenamente consciente de ese saber.

Una tercera característica del conocimiento tácito es su valor instrumental para el logro de metas en la vida diaria. En tal sentido, cuanto más se valora la meta y cuanto más directamente sostiene el conocimiento al logro de la misma, más utilizable es dicho conocimiento.

Avanzando en el tema se puede afirmar que el Conocimiento Tácito no es un sustituto de la inteligencia general (Sternberg et al., 2000). Es visto como una dimensión de la inteligencia práctica que los tests convencionales no miden adecuadamente. Como se 
La inteligencia emocional, social y el conocimiento tácito. Su valor en la vida cotidiana

sabe, los tests de CI están compuestos por problemas que han sido caracterizados como académicos o abstractos. Por tal motivo las medidas de conocimiento tácito no pueden ser un sustitutivo de la inteligencia académica. No obstante, las habilidades cognitivas generales pueden sostener la adquisición y empleo del conocimiento tácito. Como contrapartida, el conocimiento tácito no es reductible a la inteligencia académica.

Finalmente se señala que el conocimiento tácito no es suficiente para que el sujeto tenga un desempeño exitoso, ya que para que así ocurra deben entrar en juego tanto el llamado factor "g", la motivación para el logro y numerosos otros factores. Es importante tener en cuenta a todos estos a la hora de comprender el desempeño de un sujeto, tanto como de hacer intervenciones que tiendan a mejores logros en contextos de la vida real.

\section{Acerca de la Inteligencia Emocional}

Los estudios sobre la IE no tienen más de una década, es decir, son recientes en comparación con el vasto campo de investigaciones sobre la inteligencia académica. Tradicionalmente se pensó - desde una perspectiva estrecha - que el CI era genético y por lo tanto, poco modificable por la experiencia. También se consideraba que las oportunidades de éxito de un sujeto dependían de esta capacidad.

Se puede afirmar que existe un relación entre la capacidad del sujeto - evaluada por pruebas de CI - y su posterior desempeño, es decir, muchos sujetos con CI bajo se posicionan en trabajos de baja jerarquía, por ejemplo, trabajos domésticos, mientras que los de CI alto suelen ocupar cargos bien remunerados. Pero esto no es una ley infalible y se puede aseverar que el CI o las pruebas de aptitud académica son relativamente inadecuadas para predecir de un modo inequívoco quien tendrá éxito en la vida. Goleman señala que, en el mejor de los casos el CI contribuye aproximadamente en un $20 \%$ a los factores que determinan el éxito en la vida. El $80 \%$ restante estaría compuesto por otros variables inherentes a la IE, tales como: autodominio, capacidad para motivarse a sí mismo, perseverancia frente a las frustraciones, control de los impulsos, regulación del humor, empatía y tener esperanza.

Ocurre que la inteligencia académica correlaciona positivamente con el rendimiento escolar pero no habilita a resolver los problemas de la vida cotidiana. A pesar de ello, la escuela y la cultura han puesto énfasis desde siempre en las habilidades académicas y han ignorado la IE, aunque esta tenga capital importancia en el éxito en la vida diaria. Las investigaciones realizadas permiten afirmar que un sujeto con habilidades emocionales, que conoce y maneja sus propios sentimientos, que puede interpretar y afrontar con eficacia los sentimientos de otros, se posicionará con ventaja en cualquier aspecto de la vida.

\section{¿Qué es la Inteligencia Emocional?}

Es Goleman $(2000,1995)$ quien popularizó el concepto de IE y reconoce haber tomado el concepto de Peter Salovey de la Universidad de Yale. 
Psicodebate 5. Psicología, Cultura y Sociedad

Salovey señala que, en un sentido amplio la IE puede ser entendida no solo como la posesión de lo que se denomina conocimiento tácito, sino también como la habilidad para emplear ese conocimiento en la propia vida.

Goleman define a la IE como "habilidades tales como ser capaz de motivarse y persistir frente a las decepciones; controlar el impulso y demorar la gratificación; regular el humor y evitar que los trastornos disminuyan la capacidad de pensar; mostrar empatía y abrigar esperanzas"(2000, p. 54). Goleman señala que en las investigaciones acerca de los factores relacionados con la IE se destacan la empatía y la resiliencia del yo, lo que permite suponer que la IE es distinta del CI.

\section{Consideracionesteóricas acerca de la Inteligencia Emocional}

Es posible distinguir dos modelos de IE, el de las Habilidades Mentales y los Modelos Mixtos.

\section{El Modelo de las Habilidades}

En la historia de Occidente y en la psicología, las emociones y el razonamiento han sido en ocasiones vistos en oposición uno con otro. Contemporáneamente se pudo constatar que las emociones proveían información acerca del funcionamiento intelectual. Ello sugiere que emoción e inteligencia están vinculadas, tal cual lo plantean Mayer, Salovey y Caruso (2000).

Siguiendo a estos autores, la IE se refiere a la habilidad para percibir y expresar emociones con precisión, para conocerlas y comprenderlas; habilidad para emplear los sentimientos, de tal modo que faciliten el pensamiento; habilidad para reconocer el significado de las emociones y capacidad para razonar y solucionar problemas sobre la base de aquellas. Igualmente se refiere a la habilidad para regular las emociones de uno mismo y de los demás (Salovey y Pizarro, 2003; Mayer y Salovey, 1997, Mayer et al., 2000). Señalan que las habilidades emocionales se pueden dividir en 4 aspectos:

a) Percepción y expresión de las emociones

b) Empleo de las emociones para facilitar el pensamiento

c) Comprensión y análisis de las emociones

d) Manejo de las emociones (Mayer y Salovey, 1997)

1) Percibir emociones: es la habilidad de un sujeto para reconocer como se siente él mismo y cómo se sienten los que lo rodean. Implica decodificar con precisión las señales emocionales en las expresiones faciales, el tono de voz o expresión corporal. La destreza más básica es la percepción y valoración de la emociones, por ejemplo, el niño pequeño aprende el significado de esas expresiones emocionales (Mayeret al., 2000). Observa su propio llanto o alegría en el rostro de sus padres, quienes empáticamente reflejan tales sentimientos, a modo de espejo. A medida que el niño crece aprende a discriminar con más fineza entre sonrisas genuinas y las meramente formales. 
La inteligencia emocional, social y el conocimiento tácito. Su valor en la vida cotidiana

Una valoración precisa de las emociones comienza cuando se presta atención a las expresiones de los otros. Esta habilidad para percibir y valorar a aquellas es una las más importantes en la interacción social. Por ejemplo, si un sujeto no sabe cuándo un extraño le sonríe o le hace una mueca, puede quedar en una posición muy precaria. Los niños desde muy temprana edad, tanto como los sujetos de diversas culturas están capacitados para identificar las expresiones emocionales de los demás. No obstante ello, algunos carecen de esa habilidad (Saarni, 1999). Igualmente, los niños autistas y los que padecen serios trastornos del desarrollo tiene un pobre reconocimiento de las emociones de los otros.

Con respecto al conocimiento de las propias emociones Goleman señala que suele designarse como metahumor. Salovey, en cambio prefiere emplear el término consciencia de sí mismo (self-awareness), en el sentido de una atención a los propios estados internos. Salovey agrega que los pensamientos típicos que indican consciencia emocional de sí mismo, son por ejemplo, "estoy pensando en cosas buenas para alegrarme", o frente a algo perturbador "no pienses en eso"

2) Empleo de las emociones para facilitar el pensamiento: incluye la habilidad del sujeto para tener en cuenta los sentimientos cuando razona y resuelve problemas (Salovey y Pizarro, 2003). Esta habilidad consiste en determinar cómo las emociones afectan al sistema cognitivo y, de ese modo, cómo pueden ser empleadas para la toma de decisiones más efectivas. Cabe señalar que la cognición puede ser desorganizada por las emociones - como la ansiedad o el miedo - pero que tales emociones pueden contribuir a focalizar tareas cognitivas que se realizan mejor bajo particulares estados de ánimo. Los cambios en los puntos de vista sobre una cuestión pueden estar precisamente alentados por determinados estados de ánimo. De este cambio de perspectiva puede surgir el pensamiento creativo (Goodwin y Jamison, 1990; Mayer, 1986; Mayer y Hanson, 1995).

3) Comprensión y análisis de las emociones: muchos investigadores consideran que las emociones son universales, relevantes para la supervivencia y una respuesta innata a los estímulos del medio ambiente, no obstante lo cual puede afirmarse que la expresión de las emociones difiere a través de las culturas.

Se parte de la idea de que las emociones están representadas como un complejo conjunto de símbolos interrelacionados, es decir que existe un lenguaje emocional. Las destrezas incluidas aquí son la habilidad para clasificar las emociones y reconocer que existen emociones que se relacionan (Salovey y Pizarro, 2003). Comprender los signos que anteceden a diversas emociones es un componente crítico de la IE, por ejemplo, el disgusto y la irritación pueden conducir a la cólera si la causa de la irritación continúa y se intensifica. El conocimiento acerca de cómo las emociones se combinan y cambian a través del tiempo es importante para tratar con otros y para la comprensión de uno mismo.

En síntesis, tal como lo afirman Mayer et al. (2000) la IE involucra la habilidad para reconocer las emociones, para saber cómo se expresan y para actuar conforme a ello. 
Psicodebate 5. Psicología, Cultura y Sociedad

4) Manejar las emociones: éste es el más alto nivel de los aspectos que se vienen mencionando. Involucra el manejo y regulación de las emociones de uno y de los demás, tales como saber cómo calmarse luego de estar enojado, librarse de la irritabilidad, la ansiedad o la melancolía, o ser capaz de aliviar la ansiedad de otro sujeto. Salovey señala que los sujetos que son exitosos en esta esfera pueden recuperarse más rápidamente de las situaciones difíciles de la vida.

A su vez el manejo de las emociones incluye la idea de que es frecuentemente más adaptativo experienciar las emociones que reprimirlas. La regulación emocional óptima más bien va a minimizar las emociones, que eliminarlas por completo.

La habilidad para manejar las propias emociones y la de los otros es probablemente un signo de madurez y parece combinar la habilidad para percibirlas tanto como el conocimiento acerca de ellas (Salovey y Pizarro, 2003), por ejemplo la capacidad que un sujeto tiene para mantener la calma, en lugar de reaccionar con violencia, cuando una manifestación gremial corta el tráfico en una ruta que él necesita transitar. De igual modo, es posible regular los estados de ánimo de otras personas. A modo ilustrativo señalamos que si un adolescente observa que su amigo está triste porque celebra su cumpleaños, y el padre está ausente - está divorciado de la madre - buscará el modo de cambiar el tono emocional del amigo y lograr que el acontecimiento sea una experiencia grata.

En la Tabla I se esquematizan los 4 aspectos o ramas en las que pueden dividirse las habilidades emocionales y las destrezas involucradas que se conocen como el Modelo de las habilidades mentales.

Tabla 1. Inteligencia Emocional. Modelo de las habilidades mentales

\begin{tabular}{ll}
\hline RAM A & DESTREZAS INVOLUCRADAS \\
\hline Percibir emociones & $\begin{array}{l}\text { Habilidad para percibir emociones en si mismo y otros } \\
\text { tanto en objetos, arte, historia, música y otros estímulos }\end{array}$ \\
\hline Emplear las emociones para facilitar & Habilidad para generar, emplear y sentir emociones nece- \\
& $\begin{array}{l}\text { sarias para comunicar sentimientos o emplearlas en } \\
\text { procesos cognitivos }\end{array}$ \\
\hline Comprender emociones & $\begin{array}{l}\text { Habilidad para comprender información emocional, } \\
\text { cómo las emociones se combinan y para apreciar el signi- } \\
\text { ficado de las emociones }\end{array}$ \\
\hline Manejar las emociones & Habilidad para estar abierto a los sentimientosy modularlos \\
& en uno mismo y en los otros \\
\hline
\end{tabular}

El modelo de las habilidades mentales hipotetiza que es probable que un sujeto inteligente emocionalmente haya crecido en un ambiente hogareño socialmente saludable, por ejemplo, ha tenido padres sensibles. También se caracteriza por expresar las emociones eficazmente, por ejemplo, ser optimista pero con realismo; ser capaz de comunicar sentimientos, desarrollar un conocimiento acabado, de "experto", en una 
La inteligencia emocional, social y el conocimiento tácito. Su valor en la vida cotidiana particular área emocional, tal como el sentimiento estético o moral, o la resolución de problemas sociales, el liderazgo o sentimientos espirituales (Mayer \& Salovey, 1995)

\section{El Modelo Mixto de la Inteligencia Emocional}

Este modelo es sustancialmente diferente del de las habilidades mentales y ha sido propuesto por Mayer, Di Paolo y Salovey (1990) y Salovey y Mayer (1990). Si bien en la concepción de la IE hacen referencia a las habilidades mentales, también describen características de personalidad que debieran acompañar a esa inteligencia. De este modo, la IE debería distinguir a quienes son genuinos y cálidos, de aquellos que se presentan abstraídos y toscos (Mayer et al., 2000). Se consideró también que los sujetos con IE eran aquellos que generaban una cantidad mayor de proyectos, igualmente eran persistentes frente a las tareas que los desafiaban y presentaban actitudes positivas hacia la vida. Todo ello los conduce a mejores resultados y recompensas tanto para sí como para los demás (Salovey y Mayer, 1990).

Sternberg señala que luego de estas primeras teorizaciones sobre la IE se advirtió que el trabajo sería más útil si se circunscribía el concepto de IE al de habilidades mentales independientes de rasgos como la cordialidad o la sociabilidad. De este modo - manteniéndolos separados - sería posible analizar el grado en que ambos (habilidades mentales y características de personalidad) contribuían de un modo independiente al comportamiento del sujeto y a su competencia en la vida. Mayer y Salovey $(1993,1997)$ destacan que, si bien rasgos como la calidez o la persistencia son importantes, es conveniente tenerlos en cuenta como algo diferenciado de la IE.

En contraposición a esta concepción de la IE, otros teóricos expanden la significación de la IE planteando una combinación de rasgos. Tal es el caso de Bar-On (1997), quien intenta dar respuestas al interrogante de por qué algunos sujetos son más exitosos en la vida que otros. Bar-On revisó la literatura psicológica sobre las características de personalidad que se presentan relacionadas con el éxito en la vida e identificó cinco amplias áreas que incluyen:

Destrezas interpersonales: relaciones interpersonales, responsabilidad social, empatía.

Adaptabilidad: resolución de problemas, prueba de realidad, flexibilidad.

Manejo del stress: tolerancia al stress, control de los impulsos.

Estado de ánimo general (humor): alegría, optimismo.

Bar-On considera que la IE está compuesta por habilidades, competencias y destrezas que representan un conjunto de conocimientos empleados para afrontar la vida con éxito. El adjetivo emocional es empleado para enfatizar que este específico tipo de inteligencia difiere de la inteligencia cognitiva..." (Bar-On, 1997, p. 15). Combina las habilidades mentales, como la autoconciencia emocional, con otras características que se las considera separables de las habilidades mentales, tales como la independencia 
Psicodebate 5. Psicología, Cultura y Sociedad

personal, el auto observación y los estados de ánimo. Esto determina que sea un modelo mixto. Cabe aclarar que en las investigaciones no se observa generalmente una correlación consistente entre inteligencia y estados de ánimo, por ejemplo.

No obstante la envergadura de este modelo, Bar-On es cauteloso y afirma que el CI junto al cociente emocional (CE) pueden brindar una imagen más balanceada de la inteligencia general de un sujeto

Una tercera perspectiva sobre la IE fue propuesta por Goleman $(2000,1995)$, quien creó también un modelo mixto, caracterizado por cinco grandes áreas:

a) conocer las propias emociones

b) manejar las emociones

c) motivarse a sí mismo

d) reconocer las emociones en otros

e) manejar las relaciones.

Goleman - según Sternberg - estaba desplazándose conceptualmente desde la IE hacia un área más amplia. Señaló que la llamada resiliencia del yo era bastante similar al modelo de la IE, en el cual incluía competencias sociales y emocionales. Considera que este modelo mixto tiene un enorme valor predictivo a nivel de la escuela, el trabajo o la vida cotidiana.

\section{La Inteligencia Emocional y otros conceptos relacionados}

Ciertos tipos de inteligencia se definen de tal manera que se solapan con el concepto de IE, como la inteligencia personal (Gardner, 1993; 1994, 1983) y la inteligencia social (Cantor y Kihlstrom, 1987; Sternberg, 1988). De todas ellas solo la inteligencia social ha sido operacionalizada satisfactoriamente como una habilidad mental.

De los dos modelos desarrollados, el de la IE como habilidad mental se presenta como el más definido desde un punto de vista teórico (Mayer et al. 2000). Por ejemplo, si comparamos la inteligencia social con la emocional, ésta es más amplia puesto que incluye las emociones internas y privadas del sujeto que son importantes para el crecimiento personal. Por otra parte, la IE está más focalizada que la inteligencia social en los aspectos primariamente emocionales de un problema, y no en los aspectos sociales.

A su vez, los conceptos relacionados con el modelo mixto de IE son más numerosos que los vinculados con el modelo de las habilidades. Existe un vasta literatura relacionada con temas tales como la motivación, alexitimia, empatía, apertura a la experiencia, optimismo, afectividad placentera / displacentera; inteligencia práctica, autoestima, bienestar subjetivo.

\section{La Inteligencia Emocional y los aportes de Gardner}

Gardner enuncia lo que denominó inteligencias personales y admite semejanzas con el concepto de IE. 
La inteligencia emocional, social y el conocimiento tácito. Su valor en la vida cotidiana

Gardner incluye en la Inteligencia Interpersonal la capacidad para comprender a los demás, es decir qué los motiva, cómo operan, cómo trabajan cooperativamente. El núcleo de esta inteligencia es la habilidad para discernir y responder adecuadamente a los estados de ánimo, motivaciones y deseos de los demás. En tanto, la inteligencia intrapersonal es una capacidad vuelta hacia el interior del sujeto. Es la habilidad para formar un modelo preciso y realista de si mismo y ser capaz de usar ese modelo para operar eficazmente en la vida. Este tipo de inteligencia es para Gardner la clave para el acceso a los propios sentimientos, tanto como la capacidad para recurrir a ellos como orientadores del comportamiento (Gardner y Hatch, 1983).

\section{Inteligencia Emocional: Alcances y Límites del concepto}

La IE provee de un conjunto de ideas sobre los fenómenos afectivos. Va más allá del enfoque clásico de la inteligencia, incorporando el sistema emocional al estudio de aquélla y proveyendo de este modo de una teoría de las diferencias emocionales.

Se ha hecho referencia a que la IE puede ser entendida no sólo como la posesión por parte del sujeto de un conocimiento tácito, sino también de la habilidad para emplear ese conocimiento en la propia vida.

En este trabajo se han considerado dos modelos de IE y se los ha comparado. Algunas claves encontradas se pueden sintetizar en que existe un claro factor general de IE y ese factor general se bifurca en 3 subfactores específicos relacionados con la percepción, la comprensión y el manejo de las emociones (Mayer et al., 2000). Otro de los hallazgos es que la IE correlaciona de bajo a moderado con la inteligencia general.

Falta esclarecer la perspectiva popular de que existiría una entidad psicológica única e integrada que combinaría variables tales como la persistencia, el celo o la perceptividad emocional y buenas destrezas sociales. Ciertamente, no es erróneo estudiar estos factores y determinar qué aportan colectivamente para predecir algún criterio, pero tampoco es lícito designar a estas variables como una entidad única, identificada como inteligencia emocional. De lo contrario se tiene la impresión errónea de que todos estos atributos están reunidos en una unidad, cuando en los hechos son entidades más o menos independientes. Cada uno de estos elementos ha sido estudiado por separado durante muchos años sin describirse su valor predictivo.

De todo lo expuesto, Gibbs (1995) plantea si es conveniente poner tanto énfasis en el tema, en el caso de que la inteligencia emocional no sea "la verdadera medida de la inteligencia" (p. 60). La respuesta es afirmativa, en cuanto se encaren - como se viene haciendo- serias investigaciones sobre las nuevas inteligencias

Por lo tanto, existe hoy, una brecha entre la promesa y el resultado de las investigaciones que permitan estimar el valor de la IE (Davies, Stankow y Roberts, 1998; Roberts, Zeidner y Matthews, 2001). A la luz de estas críticas parece necesario poner la teoría de la IE en perspectiva, recuperando conceptos fértiles desde un enfoque psicológico y apostando a que nuevas investigaciones echarán luz sobre su valor. 
Psicodebate 5. Psicología, Cultura y Sociedad

De los dos modelos generales de IE existentes, el de las habilidades mentales, siguiendo a Mayer et al. (2000) es probablemente el único apto para denominarse IE. El otro, el mixto, es algo más general que el significado que emocional e inteligencia puede sugerir. Hasta cierto punto la suerte de las medidas de IE está vinculada a los avances en psicología de la personalidad donde se especifiquen mejores criterios de actividades diarias que incluyen éxito. De cualquier modo, es ingenuo pensar que un investigador podría dar respuestas a la pregunta de qué es una vida afectivamente exitosa.

\section{Síntesis}

La pregunta que ha orientado este trabajo ha sido qué distingue a los sujetos exitosos de los menos exitosos en la vida diaria y qué determina que sujetos exitosos en la escuela presenten déficit en sus relaciones sociales y lo inverso, que sujetos con un pobre desempeño escolar se manejen sorprendentemente bien en la vida diaria.

Las pruebas de CI no han logrado dar plena respuesta a estos interrogantes, por lo que en las últimas décadas se ha configurado un campo de investigación en torno a otras formas de inteligencia, no académicas, tanto como al esfuerzo de diseñar instrumentos que puedan evaluarlas.

Se ha hecho referencia así, a las inteligencias social, práctica, emocional y al conocimiento tácito, con el propósito de deslindar su significado y analizar sus puntos de solapamiento.

Todos estos tipos de inteligencia comparten el carácter de procesal y se centran en la solución de problemas de la vida cotidiana. Al parecer, sobre la base de lo desarrollado, la IE puede incluir con más amplitud conceptos de los demás tipos de inteligencia, de allí que se le haya destinado un amplio espacio. De los componentes que son incluidos en las definiciones de IE destacamos la habilidad para comprender a otros y para comportarse con sensatez con relación a aquellos (inteligencia social). La inteligencia práctica hace referencia a la habilidad para afrontar problemas de la vida diaria cuya solución no se encuentra disponible a partir del conocimiento adquirido en el contexto escolar. También se ha hecho referencia a que gran parte del conocimiento necesario para el éxito en las actividades de la vida diaria es tácito. Se entiende por tal a aquel obtenido de la experiencia diaria. Sternberg ha señalado que el conocimiento tácito parece ser un aspecto de la Inteligencia práctica.

Por último se ha definido y analizado la IE, donde se pueden encontrar conceptos vertidos precedentemente y una propuesta de articulación de las emociones y el razonamiento, clásicamente percibidos como opuestos. La IE hace referencia a la habilidad para percibir y expresar emociones, para comprenderlas, para reconocer el significado de las mismas y para solucionar problemas. Se han desarrollado dos modelos, el de las habilidades mentales y el modelo mixto, los cuales dejan al descubierto la disyuntiva actual frente al tema: si se trata solo de habilidades o si están implicadas variables de personalidad, tema aun abierto al debate. 
La inteligencia emocional, social y el conocimiento tácito. Su valor en la vida cotidiana

El concepto de IE - más que los de Inteligencia social y conocimiento tácito - se popularizó y, como ocurre habitualmente con las ideas que captan el interés de un gran público, se va haciendo cada vez más difícil comprender los diversos sentidos en los que es empleado el término (Mayer et al.,2000). Se complejiza así la evaluación de la misma, tanto como la determinación del valor y la utilidad de la idea original.

Es probable que el interés sorprendente que recibió este tema se vincule a la promesa sostenida por las diversas conceptualizaciones sobre la IE de que habría otro camino para ser ingenioso, agudo o para tener un buen tono emocional (Goleman, 2000).También, a que las habilidades académicas en las que la escolarización formal ha puesto tanto énfasis tienen un valor muy limitado por cuanto no han demostrado utilidad en la resolución de problemas de la vida diaria.

Por otra parte, porque se relacionó a la IE con la posibilidad de predecir el éxito en la vida de un sujeto, lo que se acompañó con afirmaciones silvestres y escritos populares de autoayuda (Mayer et al., 2000). De este modo fue vista como una especie de panacea a muchos males de la sociedad de nuestro tiempo, vinculados a disfunciones severas en el manejo de los afectos, al maltrato emocional y al comportamiento agresivo. La IE se convirtió, entonces, en sinónimo de autocontrol y atractiva personalidad, variables que no son evaluadas por pruebas estándar de CI.

Diversos temas aun no están resueltos, siendo una cuestión central si efectivamente la IE es un mejor predictor del éxito que la inteligencia general. Al respecto se ha afirmado que la IE puede predecir una variedad de comportamientos exitosos en el hogar y el trabajo, pero este planteo es incierto, puesto que no hay evidencias empíricas de que se puedan hacer tales predicciones sin margen de error. Los escritos populares sostienen que la inteligencia general predice el $20 \%$ de la varianza del éxito, mientras que la IE puede contribuir con el $80 \%$ restante. Este planteo puede resultar muy atractivo para el lector no especialista en el tema, pero lo cierto es que hasta hora no se han estudiado variables que puedan pronosticar grandes porciones de éxito, aunque ello fuera deseable.

En coherencia con Mayer et al. (2000) se puede afirmar que ese $80 \%$ inexplicable parece, en gran parte, consecuencia de la interacción compleja de ciertas variables. Por ejemplo, el éxito en la carrera profesional de un sujeto depende no sólo de componentes de personalidad, sino también de fuerzas económicas, políticas o de avances científicos.

Por lo tanto, existe hoy, una brecha entre la promesa y el resultado de las investigaciones que permitan estimar el valor de la IE (Davies, Stankow y Roberts, 1998; Roberts, Zeidner y Matthews, 2001). A la luz de estas críticas parece necesario poner la teoría de la IE en perspectiva, recuperando conceptos fértiles desde un enfoque psicológico y apostando a que nuevas investigaciones echarán luz sobre su valor.

No obstante las limitaciones señaladas, los estudios sobre el tema han tenido el mérito de ampliar nuestra concepción acerca de las habilidades cognitivas y advertir que es imprescindible integrar las emociones y los procesos de razonamiento si se quiere conocer más cabalmente el comportamiento humano. 
Psicodebate 5. Psicología, Cultura y Sociedad

\section{Referencias Bibliográficas}

Bar-On, R. (1997). The Emocional Quotient Inventory (EQI): Technical Manual. Toronto: Multi-Health Systems.

Barnes, M. y Sternberg, R. (1989) Social intelligence and decoding of nonverbal cues. Intelligence, 13: 263-287.

Cantor, N y Harlow, R. (1994) Social intelligence and personality: Flexible life-task pursuit. In R. Sternberg \& P. Ruzgis (Eds), Personality and intelligence. Cambrindge, UK: Cambridge University Press: 137-168.

Cantor, N. y Kihlstrom, J. (1987). Personality and social intelligence. Englewood Cliffs, NJ: Prentice-Hall

Davies, M. Stankow, L y Roberts, R. (1998). Emotional intelligence: In search of an elusive construct. Journal of Personality and Social Psychology, 75: 989-1015

Ford, M. E. y Maher, M. A. (1998). Self-awareness and social intelligence. In M. D. Ferrari \& R. Sternberg (Eds), Self-awareness: Its nature and development (pp 191-218). New York: Gilford Press.

Gibbs, N.(1995, October 2). The EQ factor. Time: pp. 60-63.

Goleman, (2000[1995]). La inteligencia emocional. Bs.As.: Vergara. 25a. edición

Gardner, H. y Hatch, T. (1983). Multiple Intelligences go to School, Educational Researcher, 18,8.

Gardner, H.(1993).Seven creators of the modern era. In J. Brockman (Ed), Creativity. New York: Simon \& Schuster: 28-47.

Gardner, H. (1994, 1983). Estructuras de la mente. La teoría de las inteligencias múltiples. México: Fondo de Cultura Económica.

Goodwin, F. \& Jamison, K. (1990). Manic-depressive illness. New York: Oxford University Press.

Hunt, T. (1928). The measurement of social intelligence. Journal of Applied Psychology, 12: 317-334.

Jones, K. y Day, J. (1997). Discrimination of two aspects of cognitive-social intelligence from academic intelligence. Journal of Educatonal Psychoogy, 89:486-497. 
La inteligencia emocional, social y el conocimiento tácito. Su valor en la vida cotidiana

Kosmitzki, C. y John, O. (1993). The implicit use of explicit conceptions of social intelligence. Personality \& Individual Differences, 15: 11-23.

Mayer, J. (1986).How mood influences cognition. In N. E. Sharkey (Ed.), Advances in cognitive science (pp. 290-314). Chichester, England: Ellis Horwood Limited.

Mayer, J. Di Paolo, M. y Salovey, P. (1990). Perceiving affective content in amiguous visual sitimuli: A component of emotional intelligence. Journal of Personality Assessment, 54: 772-781.

Mayer, J. \& Hanson, E. (1995). Mood-congruent judgment over time. Personality and Social Psychology Bulletin, 21: 237-244.

Mayer, J y Salovey, P. (1993). The intelligence of emotional intelligence. Intelligence, 17: 433-442.

Mayer, J. y Salovey, P. (1995). Emotional Intelligence and the construction and regulation of feelings. Applied and Preventive Psychology, 4: 197-208.

Mayer, J. y Salovey, P. (1997).What is emotional intelligence? In P. Salovey \& D. Sluyter (Eds), Emotional development and emotional intelligence: Implications for educators (pp. 3-31). New York: Basic Books.

Mayer, J, Salovey, Py Caruso, D. (2000). Emotional intelligence as Zeitgeist, as personality, and as a mental ability. In R. Bar-On \& D. Parker (Eds.), The Handbook of emotional intelligence. San Francisco, CA: Jossey-Bass: 92-117.

Moss, F. y Hunt, T. (1927). Are you socially intelligent? Scientific American, 137: 108-110.

Neisser,U. (1979). The concept of intelligence, Intelligence, 3: 217-227.

Polanyi, M. (1966). The tacit dimensions. Garden City. NY: Doubleday.

Roberts, R., Zeidner, M. y Matthews, G. (2001).Does emotional intelligence meet traditional standards for an intelligence? Some new data and conclusions. Emotion, 1: 196-231.

Saarni, C. (1999). The development of emotional competence. New York: Guilford Press.

Salovey, P. y Mayer, J. (1990). Emocional Intelligence. Imagination, Cognition and Personality, 9:185-211. 
Psicodebate 5. Psicología, Cultura y Sociedad

Salovey, P. y Pizarro, D. (2003). The value of emotional intelligence in Sternberg, R, Lautrey, J. \& Lubart, T., Models of Intelligence. International Perspectives, Washignton DC: American Psychological Association.

Schön, D. (1983). The reflective practitioner: How professional think in action. NY: Basic Books.

Sternberg, R. (1985). Beyond IQ: A triarchic theory of human intelligence: New York: Cambridge University Press.

Sternberg, R. (1988). The triarchic mind: A new theory of human intelligence. New York: Viking.

Sternberg, R. (1997). Inteligencia exitosa. Barcelona: Paidós.

Sternberg, R., Forsythe, G., Hedlund, J, Jorvath, J, Wagner, R., Williams, W, Snook, S. y Grigorenko, E. (2000). Practical intelligence in everyday life.UK: Cambridge Univesity Press.

Sternberg, R, Lautrey, J. y Lubart, T. (2003). Models of Intelligence. International Perspectives, Washington DC: American Psychological Association

Torndike, E. (1929). Intelligence and its use. Harper's Magazine, 140: 227-235.

Vernon, P. (1933). Some characteristics of the good judge of personality. Journal of Social Psychology, 4 :42-57.

Wagner, R. y Sternberg, R. (1985). Practical intelligence in real-world pursuits: The role of tacit knowledge. Journal of Personality \& Social Psychology, 49:436-458.

Wedeck, J. (1947). The relationship between personality and psychological ability. British Journal of Psychology, 36: 133-151.

Wong, C, Day, J, Maxwell, S y Meara, N. (1995). A multitrait-multimethod study of academic and social intelligence in college students. Journal of Educational Psychology, 87: 117-133. 